\title{
Pointer Teaching of C Language in Higher Vocational Colleges
}

\author{
Liping Deng ${ }^{1, a}$, Xianfang Zou ${ }^{2, b}$, Wenjuan $\mathrm{Hu}^{3, \mathrm{c}}$ \\ ${ }^{1}$ Jiangxi Vocational And Technical College of Information Application,NanChang .330043, China; \\ ${ }^{2}$ Jiangxi Vocational And Technical College of Information Application,NanChang .330043, China; \\ ${ }^{3}$ Jiangxi Normal University Science And Technology College,NanChang .330027,China;
}

a34480619@qq.com; b21337501@qq.com; '492854964@qq.com

Keywords: C language; Array;Teaching;Pointer.

\begin{abstract}
C Language Programming》 is the first programming language course which the freshman at college will get contact with.while with limited time,most of students have found it is difficult and abstract to learn this course.so the author discusses how to enable students to master the application of pointer in a shortest time.Experience has shown that introducing the computer in internal storage and the meaning of variable address when teaching the input function, or emphasizing that the array name stands for pointer teaching and learning. During the pointer teacher , using some simple or classic examples to cover all the knowledge about pointers and these have achieved good results and effects.

《C language Programming》 as an important software development introducing course.Students should be allowed to adapt and familiar with the way of thinking in computer language.And they musts focus on mastering the ability of solving problems in applying computer language,so that we able to lay a foundation for C language, "pointer" has always been a key and difficult part,impacting on students' study and understanding on some following courses.As an introducing course in software development, for most of the time,we are talking about condition,loops,arrays,functions, while the content of pointer is a necessary part in provincial,national computer level 2 and programmer exam to test.In order to achieve a better teaching results in shortest time,I sum up the teaching experience which $\mathrm{C}$ language should should pay attention to,show in the following pointers:
\end{abstract}

\section{1. the application of Input Function scanf(),and the Array Address.}

All the books introduce scanf(format control string,entry list).The entry list of this function should use " $\&$ " and the variable name. And we should put the content that we are going to input into the address of variables. Besides,id there are common character in format control string, we are required to input the common characters.Also there can be a lot of content which been input already can not be read correctly. The beginners will always feel confused and hard to understand. In fact, the continuous definition of multiple variable in memory also occupy continuous memory locations,just different types of data occupy different bytes.During the operation, users will be required to type on the keyboard as requirements when encountering the first scanf() function. And enter a lot of content. After completing the input,we should put contents into the address of the variables according to the format of the format-control string once the input is illegal, it is considered that the input comes to an end. The variables behind will not get the result. For example, 
scanf(“\%d:\%d”,\&a,\&b); if you enter $3,4 \swarrow$, a gets the value of 3 ; the input format string has required to enter “:”,while you enter “,” that is obviously not legal, so the input action of scanf function ends, the latter variable b can not take the result. At the same time, it does a good job in front of the input function, if there are multiple scanf function, to ensure that each input is legal and effective.

The Array name stands for the first address of the array,so in the scanf() function, the corresponding input list of the format string which uses "\%s" do not need to add "\&".

For example:

char str[20]

Scanf(“\%s",str); /*store the content into the address where the array first address begins*/ printf(“\%s”,str); /*output the content of the arrays first address*/

These contents are actually related to computers real storage (That's say it is related to the address)

\section{Pointer}

Pointer is the address. In fact, we have introduced some application relating to the address when we are talking about the input and output functions and arrays.Students will not be much afraid of pointer when they know that they have learned of used before.

\subsection{The concept of pointer}

Pointer is the memory address,Here we must first distinguish three similar concepts:name, address and content(value). Name is used to describe the memory space which is easy to each in memory has a number, that is the address,the number in the corresponding memory unit address is the content or value.

To help reader to understand links and differences between the three concepts, we might make an analogy. There is a teacher office building, each room is assigned to the corresponding functional department, each room hangs a name:electronic etc, if the electronic department is allotted to room 101, we have to find the teacher(content) from the major or we can also search for electronic department or search for room 101.(According to the address ). Similarly, for accessing to a storage space, we can not only point out it's name but also it's address.

In $\mathrm{C}$ language. If the content of variable $\mathrm{p}$ is another variable $\mathrm{i}$ is address, that's say variable $\mathrm{p}$ is pointing to variable $\mathrm{i}$ or $\mathrm{p}$ is regarded as a pointer which is pointing to the variable $\mathrm{i}$. It is shown in picture 1



We can draw a conclusion from this: the pointer is the address and the variable storing other variable's address is a pointer variable.Pointer variable is a variable which also has three key elements.But it is a special variable. It's particularity reflected in the values.

In details:

(1) variable name: Is the same as the general variable naming rule.

(2) The value of the variable :Is the memory address of a variable.

(3) Variable type:Is mainly the type of the variable that it points to.

Pointer is very flexible. It can not only point to a simple variable, but also pointer to a array, function, also can be used as a function's parameter.

2.2 Pointer to simple variable.

For example: 


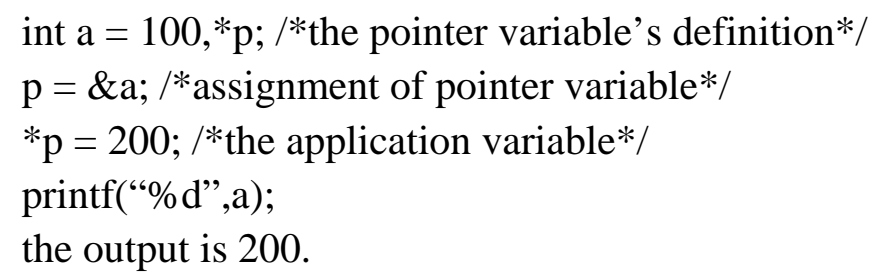

Here we have to understand once we define a pointer and the pointer points to simple variable. Excepting the defined $\mathrm{p}$ and the initialized $\mathrm{p}$, other ${ }^{*} \mathrm{p}$ are equivalent to the simple variable it points to $\left({ }^{*} \mathrm{p}<=>\mathrm{a}\right)$; ${ }^{*} \mathrm{p}$ is equivalent to the address of the simple variables. $(\mathrm{p}<=>\& \mathrm{a})$. The statement above can be changed to output:printf(“\%d”, *p);

2.3 Pointer to arrays.

2.3.1 Pointer to a one-dimensional array.

For example:

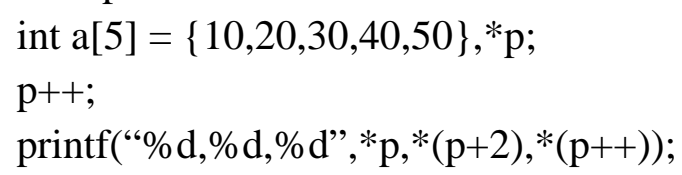

The output results are as follows: 30, 50, 20

The content above is the basic application of one-dimensional first, we have to understand the operation of the pointer

\&: The address operator.

* : Pointer operator (inter-access operator),take the value that pointer pointing to, if it points to two-dimensional array get a step closer to take the value.

++ : Arithmetic operator, representing the pointer move down or right for one unit.(Unit is related to specific data types, if it is an integer, the unit refers to two bytes, real number refers to 4 bytes).

--: Arithmetic operator representing pointer move upper or left for one unit . If we define a pointer and it points to one-dimensional array, the $\mathrm{p}<=>\mathrm{a}$ can shown as ${ }^{*} \mathrm{p}<=>* \mathrm{a}$, $*(\mathrm{p}+\mathrm{i})<=>\mathrm{a}[\mathrm{i}]<=>\mathrm{p}[\mathrm{i}]<=>*(\mathrm{a}+\mathrm{i})$. But attentively. $\mathrm{P}$ and a are address but also different. $\mathrm{P}$ is the variable address, $\mathrm{a}$ is a constant address. There can be a $\mathrm{p}^{++}$rather than $\mathrm{a}++$.

2.3.2The meaning of the array pointer and the array of pointers.

The former is a pointer, the latter is an array.

(1)Definition format:just a couple of parentheses.

(1): The pointer to a one dimensional array. Supposing a defined statement: int $\left({ }^{*} \mathrm{p}\right)[4]$;

(2):pointer array

Supposing a defined statement :int *p[4];

(2)Application occasions: the former is pointer and the latter means multiple pointers.

(1): The pointer to a one-dimensional array.

In general, we assign a two-dimensional array name to a pointer which is pointing to a one-dimensional array to access a row in two-dimensional array.

(2): Pointer array.

It is suitable for for storing a several strings to make the management of character string more flexible.Character pointer array processing string can not only save memory, but also can improve the efficiency of the operation. 


\subsection{Pointers to function}

2.4.1 Pointer as a function of the parameter

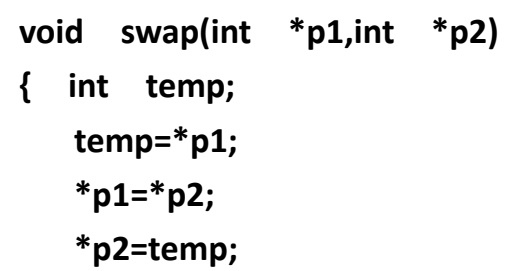

After exchange in the left, the values of the parameters can be passed to the argument, because the values of the pointer is pointing to have exchanged. While on the right only the pointer to pointer changed, the original value not exchanged.

\subsubsection{The pointer function and the function pointer.}

They are tow completely pointers with different concepts. The former is function and the latter is pointer.

(1) Definition format: just a couple of parentheses.

(1) Pointer function

Supposing there is a defined statement

int $*$ fp(int $\mathrm{x}$, int $\mathrm{y})$;

(2) The pointer to functions.

Supposing there is a defined statement:

int $(* \mathrm{fp})($ int $\mathrm{x}$, int $\mathrm{y})$;

(2) Application occasions:

The former is function, is only a function of the return value is a pointer, the latter is a pointer. Which points to a function. Through this pointer, the function can be called indirectly.

\section{3 . Summary}

The paper discusses that in the pointer teaching process, by intercepting the most basic and most important knowledge to analysis of its application problems, the purpose is to enable students to master the basic knowledge in a relatively short period of time. which is expected to be one of the effective methods of pointer teaching.Is expected to be one of the effective methods of teaching.

\section{References}

[1] Guan YinZhi. C language programming examples tutorial [M]. Beijing; people's Posts and Telecommunications Press, 2011

[2] Tan Haoqiang. C programming (Second Edition) [M]. Beijing; Tsinghua University press, 2001

[3] BaiYu. C language tutorial [M]. Beijing; Electronic Industry Press, 2009 